Eighteenth General Hospital, early on the 29th, there was present a typical gas odor, bubbles could be expressed on slight pressure, and tissue crepitus was noticed. The entrance and exit wounds in the scapular region were connected by a long incision. Necrotic gaseous supraspinatus and infraspinatus muscles with much of the bone were excised, and Carrel's tubes were laid in for irrigation. By the 30th the wound appeared well, and suppuration was beginning under one edge. Suddenly, November 1, his temperature, which had dropped below 100, jumped to 103.4, and on the 4th he passed away with a lobar pneumonia. There was no further evidence of gas infection in the wound.

\section{THE EPIDEMIC OF MENINGITIS AT CAMP JACKSON}

\section{PRELIMINARY REPORT}

W. W. HERRICK, M.D.

Major, M. R. C., U. S. Army; Chief of Medical Service, Base Hospital, Camp Jackson, Columbia, S. C. NEW YORK

The epidemic of meningitis at Camp Jackson will be reported in detail at a later time. It seems profitable to bring to general notice some of the peculiar features of the epidemic and some of the methods used to arrive at an early diagnosis, along with certain modifications of treatment that have proved valuable.

Over 140 cases have been observed and classified as abortive, ordinary and fulminating. The last have generally shown extensive purpura, with death in from four to ten hours, without the clinical or necropsy findings of meningitis.

The disease is not primarily a meningitis. It is a generalized systemic invasion by the meningococcusa sepsis - with possible secondary involvement of the meninges, joints, pericardium, endocardium, testicles, conjunctiva, sclera, pleura, lungs, from all of which regions, in addition to the tonsils and pharynx, the micrococci have been isolated. In many cases the systemic symptoms appear from twenty-four to seventytwo hours before involvement of the meninges. These general symptoms are not at all characteristic. Moderate fever, weakness, mild apathy, coated tongue, pharyngitis or coryza, and mild digestive disturbances are most common. Headache and other cerebral symptoms are generally absent at this stage.

In about 75 per cent. of the cases in this epidemic, the earliest sign of value in diagnosis has been the petechial rash. In order of frequency this rash appears on the deltoid regions, hips, trunk, extremities, mucous membranes, and face. The purpura of the fulminating cases does not originate in these petechiae, but is apparently a separate lesion.

The meningococcus can usually be recovered in the spinal fluid withdrawn at the first or second lumbar puncture, and often from six to thirty-six hours before the characteristic clinical picture develops or cellular increase and cloudiness occur in the fluid. It is our impression that the cerebral precedes the spinal involvement. For this reason, in dotbtful cases, the lumbar puncture should be repeated at intervals of from three to six hours in order to drain the meningococcus from the brain. Thorough centrifugation and careful examination of the sediment usually reveal one or more pairs of meningococci. At this early stage these organisms are extracellular and variable in size and appearance. The spinal fluid is clear, has a normal cell content, without polymorphonuclears, usually reduces Fehling's solution, and may or may not show a faint trace of globulin.

Diagnosis in the stage of meningococcic sepsis may be made many hours before the meningococcus has time to develop its characteristic selective action on the meninges. In the present epidemic, a rapidly developing petechial rash, fever and variable general symptoms and the findings of isolated meningococci in the spinal fluid have been the important factors in an early diagnosis.

The term "epidemic cerebrospinal meningitis" is misleading when applied to an epidemic of this character. "Spotted fever" is more satisfactory, but inadequate because a minority of the patients show little or no rash. "Meningococcus sepsis" is more correct.

In the light of the foregoing observations, the usual methods of treatment have been modified, and with success. In the stage of sepsis before meningitis has developed, routine intravenous administration of meningococcus antiserum in doses of 20 to 60 c.c. has been effective and has in many cases been followed by striking improvement. Such doses have been administered every twenty-four hours during the first three or four days of the disease. We feel that intravenous serotherapy should precede the intraspinal, and that in these cases of primary meningococcus sepsis it is probable that such intravenous serum administration is of more importance than the ordinary intraspinal treatment. The latter must not be neglected, but is probably best deferred in some cases until the spinal fluid becomes cloudy. It has been pointed out by Amoss and his co-workers that the injection of serum intrathecally increases the permeability of the choroid plexus for the virus of poliomyelitis. It is important that the same question be experimentally investigated in meningococcus sepsis.

\section{INTERPRETATION OF THE SCHICK REAC- TION IN RECRUITS FOR THE NATIONAL ARMY*}

\author{
ABRAHAM ZINGHER, M.D., NEW YORK \\ First Lieutenant, M. R. C., U. S. Army \\ CAMP UPTON, YAPHANK, N. Y.
}

Occasional outbreaks of diphtheria in military camps, and the possibility of preventing them by utilizing the more recent advances in the prophylaxis against this disease, have caused us to apply the Schick test to the recruits for the National Army on their admission to Camp Upton, Yaphank, L. I. , Over 2,500 men have thus far been tested. Owing, however, to the assignment of the recruits, within forty-eight hours, to different regiments scattered over the camp, it was found necessary to discontinue making the test at the time of the primary physical examination. The test will be applied later, when it can be more conveniently done, to all the members of each regiment.

During the past four years an increasing number of hospitals and various institutions have adopted the Schick test as a routine procedure for determining the susceptibility or immunity of children and adults to diphtheria. In children the test is simple and extremely reliable, if properly made with a well ripened toxin. The pseudoreaction is only occasionally seen in children, but in adults it is frequently

* Presented before the Section on Medione, New York Academy of Medicine, Oct. 16, 1917. 